The surface brightness distribution along an east-west path through the Trapezium was measured at three wavelengths within the blended emission component. The observed brightness distribution agrees to within 20 per cent with the distribution of emission measure calculated from a spherically-symmetrical electron-density model derived from $[\mathrm{O}$ II $] \lambda_{3729} / \lambda_{3726}$ intensity ratios by Osterbrock and Flather, and with the brightness distribution including reddening corrections derived from $\mathrm{H} \beta$ flux measurements by Mendez. This agreement suggests that the core may be assumed optically thin in first approximation.

It is suggested that the observed contours and intensities of the $\lambda_{1} 0830$ line can be quantitatively interpreted by assuming that self-absorption by $\operatorname{HeI}\left(2^{3} \mathrm{~S}\right)$ is taking place in an expanding shell surrounding the Nebula. In particular, the required velocity distribution of $\mathrm{HeI}\left(2^{3} \mathrm{~S}\right)$ is found to be consistent with the envelope of interstellar $\mathrm{HeI}_{1} \lambda_{3} 889$ absorption observed in the spectra of the Trapezium stars. It is estimated that about 70 per cent of the $\lambda$ ro830 photons originally emitted by the bright, but optically thin, core of the Nebula, are deleted from the line of sight by scattering in the expanding zone. The apparent optical depths at $\lambda$ ro83o are consistent with those at $\lambda_{3} 889$ provided secondary scattering in the expanding zone is taken into account.

\title{
9. THE TIME SCALE OF THE NEBULA
}

\section{P. O. Vandervoort}

In a discussion of the 'time scale' of the Orion Nebula one is primarily interested in the age of the Nebula or, more precisely, in the interval of time that has elapsed since the radiation in the Lyman continuum of the exciting stars $\theta^{1}$ Ori $\mathrm{C}$ and $\theta^{2}$ Ori A first ionized and heated the gas. Presumably, this time scale also refers to the ages of the exciting stars and of the stars in addition to $\theta^{1}$ Ori $\mathrm{C}$ which are members of the Trapezium. The age is derived from the comparison of a gas-dynamical model of the Nebula with observational results concerning the distributions of the density and the velocity of expansion of the ionized gas.

The interpretation of the relevant observational data must be based on a knowledge of the geometry of the Nebula. According to Osterbrock and Flather (r), Menon (2), Wilson, Münch, Flather, and Coffeen (3), and others, it appears that the Nebula is approximately spherically symmetric about the Trapezium. Menon (2) has derived a spherically symmetric model of the distribution of the electron density from the distribution of the surface brightness at a wavelength $3.75 \mathrm{~cm}$. In their investigation of the Doppler shifts and widths of the spectral lines $\mathrm{H}_{\gamma}, \lambda_{3726}[\mathrm{OII}]$, and $\lambda_{5007}$ [O III], Wilson et al. found evidence that the Nebula is expanding with a velocity of approximately $10 \mathrm{~km} / \mathrm{sec}$ in an inner region where the lines of [O III] originate and a velocity of approximately $7 \mathrm{~km} / \mathrm{sec}$ in an outer region where the lines of [OIr] originate. The precise locations of these regions are not known; however, since the lines of [O III] are very faint at distances from the Trapezium greater than $5^{\prime}(\mathrm{I})$, it can be argued that the observed radiation in these lines originates within the corresponding linear distance from the Trapezium.

The construction of a gas-dynamical model of the Nebula is made difficult by the characteristic feature of the evolution of an HII region that the state of motion of the ionized gas is determined by the initial state of the cloud of neutral gas in which the Hrr region forms and by the manner in which the exciting stars begin to radiate in the Lyman continuum (4). After the onset of the ionizing radiation, an $\mathrm{HII}$ region grows as the ionization front separating the 
ionized gas from the surrounding neutral gas propagates outward from the exciting stars. The neutral gas overrun by the front is ionized and set into motion. The motion of the ionization front and the density and velocity of expansion with which the ionized gas is left by the front are determined by the flux of ionizing radiation reaching the front and by the state of the neutral gas ahead of the front. The subsequent motion of the ionized gas is determined by the gradient of the pressure. Mathematically the construction of a model of an Hir region requires the solution of this initial value problem governing the motion of the gas. Therefore a careful consideration of the initial conditions appropriate to the Orion Nebula is required.

Kahn and Menon (5) have derived an age of the order $10^{4}$ years from a comparison of Menon's observational model of the distribution of the electron density with Goldsworthy's similarity solution for the distribution of the density in an expanding HII region (6). The similarity solution contains a shock in the ionized gas at a radial distance from the centre which is a determinate function of the time, and behind the shock the density falls to a relative minimum at the centre. Neither a shock nor a central region of low density is resolved in Menon's observations. From this negative result Kahn and Menon derive an upper limit on the radius of the shock and a corresponding upper limit on the age of the Nebula. However, the occurrence of the shock in the similarity solution is a consequence of unphysical initial conditions in which the initial distribution of the neutral gas is singular at the origin and the onset of the ionizing radiation is instantaneous. On the other hand it is known that no shock appears in the solution of the equations governing the motion of the ionized gas in an HII region when the initial density of the neutral gas is finite at the origin and the onset of the ionizing radiation is instantaneous. It is not known whether or not a shock would appear if the finite time scale for the onset of the ionizing radiation were taken into account. But if such a shock were present in the Orion Nebula, it is unlikely that its motion would be accurately described by the similarity solution. For these reasons the validity of this estimate of the time scale cannot be regarded as established.

It appears that a more reliable estimate of the age of the Orion Nebula is obtained with the help of a model constructed for initial conditions in which ( $I$ ) the neutral gas is initially at rest with a distribution of the density similar to the present distribution of the density of the ionized gas, and (2) the interval of time required for the exciting stars to begin radiating with their present luminosities in the Lyman continuum is negligible (7). (For convenience the isothermal distribution was chosen to represent the initial distribution of the neutral gas, but it was not intended that any physical significance should attach to this choice.) After the onset of the ionizing radiation an interval of time of the order of 300 years is required for the ionization and heating of the gas within $3 \mathrm{pc}$ of the centre of the model. The motion of the ionization front is highly supersonic, and the ionized gas is initially left approximately at rest with the distribution of density that prevailed in the neutral gas. Once the gas is ionized and heated the model expands isothermally. At a reasonably well defined stage of its evolution the model has a distribution of the density which is in satisfactory agreement with Menon's model. At about this same stage the velocity of expansion has attained a value of the order of $10 \mathrm{~km} / \mathrm{sec}$ in the inner part of the model (at a radial distance from the centre of about $5^{\prime}$ of arc) and somewhat smaller values in the outer parts. This distribution of the velocity seems to represent the results of Wilson et al. satisfactorily. The age at which the model represents the present state of the Nebula is of the order $1 \cdot 4-2 \cdot 3 \times 10^{4}$ years if the value $45^{\circ} \mathrm{pc}$ is adopted as the distance of the Nebula from the Sun. A somewhat better representation of the present state of the Nebula might have been obtained if the initial distribution of the neutral gas had been chosen to have a steeper gradient and a higher density in the region within $5^{\prime}$ of the centre. However, the present estimate of the age of the Nebula would not be significantly changed by this modification (7).

The estimated age of the Nebula is comparable with the Helmholtz-Kelvin time scales for the contraction of the exciting stars, so the time scale for the onset of the ionizing radiation was 
probably not negligible during the evolution of the Nebula. Since the luminosities of the exciting stars in the Lyman continuum must have increased to their present values at a finite rate, the ionization of the Nebula must have occurred more slowly than is indicated by the model. At the correspondingly lower velocity the ionization front would have left the ionized gas with a finite velocity of expansion and a distribution of the density which would have differed from the initial distribution in the neutral gas. These effects should give rise to some quantitative differences between the Nebula and the model. And if the onset of the ionizing radiation was sufficiently slow, the early evolution of the Nebula must have been even qualitatively different from that of the model in the sense that in the Nebula a shock must have preceded the ionization front into the neutral gas. Investigations of the dependence of the early evolution of an $\mathrm{H}$ II region on the time scale for the onset of the ionizing radiation have been undertaken only recently $(8,9)$, and it is not yet known to what extent such considerations will require a modification of the present model of the Nebula.

Although the model may give an overly simplified representation of the evolution of the Nebula, it should suffice to determine the age of the Nebula quite reliably. The essential characteristic of the model is that it represents the Nebula as a cloud of gas that expands freely from an initial state of rest. From the computed structure and evolution of the model one may conclude that when such a cloud has a temperature and a distribution of the density similar to those prevailing in the Nebula, the gas acquires a velocity of expansion of the order of the observed value $10 \mathrm{~km} / \mathrm{sec}$ in an interval of time of the order $2 \times 10^{4}$ years. This must be the order of the age or an upper limit on the age, since we may be moderately certain that the ionized gas has been expanding from an initial state of rest or expansion. To an adequate approximation the original cloud of neutral gas may be considered to have been at rest or in expansion. The neutral gas might have been collapsing in the prevailing gravitational field, but the velocity of collapse could hardly exceed the velocity of escape from the Orion Nebula Cluster which is about $2 \mathrm{~km} / \mathrm{sec}$. And quite generally the process of the ionization of the gas would tend to impart to the ionized gas an initial velocity of expansion.

The assumption that only the gradient of the pressure exerts an appreciable force on the ionized gas requires some justification. The gravitational force in the Nebula and Cluster is not more than about 2 per cent of the force arising from the pressure (7). Although the possibility that the expansion of the Nebula is inhibited by an external magnetic field cannot be excluded, such inhibition would be significant only if the field had an unreasonably high strength of the order $8 \times 1^{-5}$ gauss (ro). The turbulent pressure arising from the random motions of the ionized gas (3) has some effect on the expansion of the Nebula, increasing the acceleration of the gas and reducing the time scale by about 15 per cent $(7)$.

Since the age of the Nebula is a measure of the age of the exciting stars, it seems to be established that the members of the Trapezium $\theta^{2}$ Ori $A$, and perhaps other stars in the region have formed much more recently than most of the members of the cluster. This interpretation is consistent with the age of the Trapezium of ro $^{4}$ years derived by Parenago (Ir) from the relative motions of the stars. However, the Helmholtz-Kelvin time scales for the contraction of these stars vary from values of the order $10^{4}$ years for $\theta^{1}$ Ori $\mathrm{C}$ and $\theta^{2}$ Ori $\mathrm{A}$ to values in excess of $10^{5}$ years for the B-type stars in the Trapezium, and it is difficult to understand how all of these stars can have arrived on or near the main sequence simultaneously to within an uncertainty of about $2 \times 10^{4}$ years. Even if the initial contraction of such massive stars is of the Hayashi type, the total time scale for contraction to the main sequence is still of the order of the Helmholtz-Kelvin time scale (12). It appears that a discrepancy exists unless either the B-type stars began to contract some $10^{5}$ years or so before the O-type stars or the HelmholtzKelvin time scales are not relevant to the formation of these stars. In connection with the latter possibility, an attractive hypothesis is that the members of the Trapezium were formed 
in a single event governed by a common time scale. But even along these lines it may be difficult to account for the simultaneous formation of the Trapezium and $\theta^{2}$ Ori A.

Apart from being used to establish the bare time scale, a gas-dynamical model may be used to investigate other aspects of the evolution of the Nebula. For example, the initial distribution of the neutral gas in the present model is roughly the distribution that would have prevailed in the unionized Nebula if the gas had been in hydrostatic equilibrium at a temperature $1-3 \times$ $10^{2}{ }^{\circ} \mathrm{K}$ in the prevailing gravitational field of the cluster $(7)$. (Since the masses of the Nebula and cluster are approximately $\mathrm{IO}^{2} \odot$ and $\mathrm{I}-3 \times 1 \mathrm{O}^{3} \odot$, respectively, it is the cluster that determines the local gravitational field $(2, \mathbf{1 3})$.) Thus the gas may be regarded as having been trapped in the gravitational potential well of the cluster. This picture provides a natural explanation of the similarity of the distributions of the gas and stars that has been noticed previously $\left(\mathbf{1} 3, \mathbf{1}_{4}\right)$. Of course departures from hydrostatic equilibrium must have occurred as a result of temporal and spatial fluctuations of the field. For this reason and for the reason that there are uncertainties connected with the problem of the finite time scale for the onset of the ionizing radiation, this picture is only tentative. Nevertheless, this example illustrates the potential usefulness of a gas-dynamical model in the reconstruction of the evolution of the Nebula.

\section{REFERENCES}

I. Osterbrock, D. E., Flather, E. Astrophys. F., I29, 26, I959.

2. Menon, T. K. Publ. nat. Radio Astr. Obs., I, no. I, I96r.

3. Wilson, O. C., Münch, G., Flather, E., Coffeen, M. F. Astrophys. F. Suppl., 4, 199, I959.

4. Vandervoort, P. O. Astrophys. $\mathcal{F}$., 137, 381, 1963.

5. Kahn, F. D., Menon, T. K. Proc. nat. Acad. Sci., 47, 1712, 1961.

6. Goldsworthy, F. A. Phil. Trans. R. Soc. London, A253, 277, 196r.

7. Vandervoort, P. O. Astrophys. F., 139, 869, 1964.

8. Mathews, W. G., Bodenheimer, P. Astr. F., 69, 552, I964.

9. Vandervoort, P. O. Unpublished.

ro. Vandervoort, P. O. Astrophys. F., 138, 294, 1963.

II. Parenago, P. Astr. Zu., 30, 249, I953.

12. Hayashi, C. Publ. astr. Soc. Fapan, 13, 450, I961.

13. Johnson, H. M. Publ. astr. Soc. Pacif., 73, 147, 1961.

14. Menon, T. K. Astrophys. F., 136, 95, 1962.

\section{DISCUSSION}

H. C. Van de Hulst. But is the Orion Nebula density-bounded or not? If it is, any reference to $\mathrm{HI}$ would be irrelevant.

T. K. Menon. My conclusion regarding the density boundedness of the Nebula is based on the comparison of the observed $\int N_{\mathrm{e}}^{2} r^{2} d r$ with Strömgren's theory.

K. Wurm. Spectra of the Orion Nebula show [OIII] emission clearly up to the edge. This speaks clearly in favour of a mass-bounded Nebula: there can be no mass beyond the border.

$S$. Pottasch. I can see no good argument as to why it must be density bounded. The fact that [OrI] is observed to the extreme outer boundary, mentioned by Dr Wurm, is not inconsistent with it being an ionization-bounded region, since radiation capable of ionizing $\mathrm{O}^{+}$will certainly extend to the edge. In this connection, the application of Zanstra's method to determine an ultraviolet temperature under the assumption of an ionization-bounded volume, gives a temperature that is consistent with the spectral type O6 of the exciting stars, and with temperatures obtained from other exciting stars in other diffuse nebulae.

$K$. Wurm. But the application of Zanstra theory is too uncertain, because the temperature of the star is too inexactly known. 
R. D. Davies. High-resolution studies at Jodrell Bank show the existence of neutral hydrogen in a ring of diameter $30^{\prime}$ surrounding the Orion Nebula. The mass of this material is $150 \odot$, and the density within the shell is $\mathrm{I}_{7}$ (neutral) $n_{\mathrm{H}} \mathrm{cm}^{-3}$.

$H$. van Woerden. There does appear to be neutral hydrogen close to the Nebula in space. It may be that the ionizing radiation is partly shielded by dense clumps or filaments of neutral $\mathrm{H}$, and that part of the radiation leaks out of holes between.

G. Münch. The real problem related to the age of the Orion Nebula is not how long it has been an expanding Hir region, but rather how a very massive complex with density exceeding $10^{4}$ atoms $\mathrm{cm}^{-3}$ managed to exist for the age of the Galaxy, notwithstanding the disruptive effects of tidal forces, pressure gradients, supernova explosions, and so forth. Or in other words, where in the sky is the complex which will produce a similar nebula to Orion about $10^{7}$ years from now?

\section{INHOMOGENEITIES IN THE ORION NEBULA}

\section{F. D. Kahn}

Determinations of the (electron) density in the Orion Nebula seem really to lead to an evaluation of $\left(\overline{n^{2}}\right)^{1 / 2}$, for any given region. In deducing $\bar{n}$ from $\left(\overline{n^{2}}\right)^{1 / 2}$, one usually forgets that there may be important small-scale fluctuations in the density $n$, and so one overestimates $\bar{n}$. Perhaps this is how the inference has arisen that there is such a violent density and pressure gradient in the Nebula, which then results in calculations such as Dr Vandervoort's in a short predicted lifetime. In reality, this short lifetime may simply be due to these being more pronounced density fluctuations near the centre of the Nebula. Dr Menon has already given reasons why these might have to be postulated in any case.

A possible origin of such fluctuations is as follows: consider a collection of globules of nonionized gas, each held together by self-gravitation. On exposure to Lyman continuum radiation, each globule surrounds itself with a bright rim of ionized gas, which expands outward. The incident radiation has to keep this gas ionized, and in this way the neutral gas is almost, but not entirely, shielded from the radiation. This may be expressed by the relation

$$
\mathcal{y} \cong \beta \int_{R}^{\infty} n^{2} d r
$$

where, for simplicity, spherical symmetry is assumed. The motion of the ionized gas is governed by the equation of continuity

and by Bernouilli's equation

$$
F=n u r^{2}
$$

$$
\frac{1}{2} u^{2}+a^{2} \log \frac{n}{n_{0}}=\frac{1}{2} a^{2}
$$

wherein

$\beta=$ the recombination coefficient $\left(\cong \mathrm{IO}^{-13} \mathrm{~cm}^{3} \mathrm{sec}^{-1}\right)$,

$\mathcal{f}=$ incident flux in the Lyman continuum ( $\cong{ }_{10}^{12}$ photons $\left.\mathrm{cm}^{-2} \mathrm{sec}^{-1}\right)$,

$a=$ isothermal speed of sound in the ionized gas $\left(\cong 10^{6} \mathrm{~cm} \mathrm{sec}^{-1}\right)$,

$n_{0}=$ density of ionized gas at ionization front,

$R=$ radius of ionization front. 